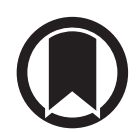

CrossMark

\title{
Treatable traits: a new paradigm for 21st century management of chronic airway diseases: Treatable Traits Down Under International Workshop report
}

\author{
Vanessa M. McDonald ${ }^{1,2}$, James Fingleton $\mathbb{1}^{3,4}$, Alvar Agusti ${ }^{5}$, Sarah A. Hiles ${ }^{1}$, \\ Vanessa L. Clark ${ }^{1}$, Anne E. Holland $\mathbb{1}^{6}$, Guy B. Marks ${ }^{7,8}$, Philip P. Bardin ${ }^{9}$, \\ Richard Beasley (10) ${ }^{3,4}$, lan D. Pavord ${ }^{10}$, Peter A.B. Wark ${ }^{1,2}$ and Peter G. Gibson (1) ${ }^{1,2}$ \\ on behalf of the participants of the Treatable Traits Down Under International \\ Workshop
}

Affiliations: ${ }^{1}$ Priority Research Centre for Healthy Lungs and Centre of Excellence in Severe Asthma, Faculty of Health and Medicine, University of Newcastle, Newcastle, Australia. ${ }^{2}$ Dept of Respiratory and Sleep Medicine, John Hunter Hospital, Newcastle, Australia. ${ }^{3}$ Respiratory Medicine Dept, Capital and Coast District Health Board, Wellington, New Zealand. ${ }^{4}$ Asthma and COPD Programme, Medical Research Institute of New Zealand, Wellington, New Zealand. ${ }^{5}$ Respiratory Institute, Hospital Clinic, Universitat de Barcelona, IDIBAPS, CIBERES, Barcelona, Spain. ${ }^{6}$ Discipline of Physiotherapy, La Trobe University Dept of Physiotherapy, Alfred Health, Institute for Breathing and Sleep, Melbourne, Australia. ${ }^{7}$ South Western Sydney Clinical School, UNSW, Sydney, Australia. ${ }^{8}$ Woolcock Institute of Medical Research, The University of Sydney, Sydney, Australia. ${ }^{9}$ Lung and Sleep Medicine, Monash University and Medical Centre, Clayton, Australia. ${ }^{10}$ Respiratory Medicine Unit and NIHR Oxford Respiratory BRC, Nuffield Dept of Medicine, University of Oxford, Oxford, UK.

Correspondence: Vanessa M. McDonald, Priority Research Centre for Healthy Lungs and Centre of Excellence in Severe Asthma, Faculty of Health and Medicine, University of Newcastle, Level 2 West Wing, 1 Kookaburra Circuit, New Lambton Heights, NSW 2305, Australia. E-mail: vanessa.mcdonaldanewcastle.edu.au

@ERSpublications

Treatable traits are proposed as an approach to personalised medicine for people with airway diseases. This review highlights the essential components of this approach, and how we can progress research and implementation. http://ow.ly/gV9I30nMHQ1

Cite this article as: McDonald VM, Fingleton J, Agusti A, et al. Treatable traits: a new paradigm for 21st century management of chronic airway diseases: Treatable Traits Down Under International Workshop report. Eur Respir J 2019; 53: 1802058 [https://doi.org/10.1183/13993003.02058-2018].

ABSTRACT "Treatable traits" have been proposed as a new paradigm for the management of airway diseases, particularly complex disease, which aims to apply personalised medicine to each individual to improve outcomes. Moving new treatment approaches from concepts to practice is challenging, but necessary. In an effort to accelerate progress in research and practice relating to the treatable traits approach, the Treatable Traits Down Under International Workshop was convened in Melbourne, Australia in May 2018. Here, we report the key concepts and research questions that emerged in discussions during the meeting. We propose a programme of research that involves gaining international consensus on candidate traits, recognising the prevalence of traits, and identifying a potential hierarchy of traits based on their clinical impact and responsiveness to treatment. We also reflect on research methods and designs that can generate new knowledge related to efficacy of the treatable traits approach and consider multidisciplinary models of care that may aid its implementation into practice. 


\section{Introduction}

In the early and mid-20th century, pharmacotherapy was driven by Paul Ehrlich's zauberkugel (magic bullet) theory and best characterised by the use of antibiotics for infectious diseases, where a single drug could precisely target the infectious agent causing the disease, resulting in cure. The latter half of the 20th century saw a dramatic rise in the burden of noncommunicable diseases (NCDs), such as cardiovascular disease, type 2 diabetes mellitus, cancer and chronic airway diseases, where the zauberkugel approach could not be usefully applied because a single aetiological agent could not be identified. A step-therapy approach was then developed to treat these conditions where drugs were added (stepped-up) or withdrawn (stepped-down) based on the risk, severity and level of responsiveness to treatment. Step-therapy has become a dominant treatment paradigm for NCDs, particularly in airway disease. This is characterised by the stepwise escalation of long-acting bronchodilators and/or inhaled corticosteroids. While this approach significantly improved asthma outcomes, in the early 21st century its limitations have become apparent and improvements in airway disease outcomes have now stalled [1,2]. Individual variability in both clinical presentation and treatment response is increasingly recognised, but whether this is systematically addressed is questionable. Furthermore, the pathway for development of impactful new treatment approaches has been haphazard, typically failing to recognise the advantage of targeting treatments [3].

In response to the limitations of step-therapy, the treatable traits approach has emerged. "Treatable traits" is a new strategy where patients are individually assessed for a specified set of treatable problems, and an individualised treatment programme is developed and implemented based on this multidimensional assessment. This new paradigm of disease management offers great promise in individualising care and improving outcomes for patients with airway diseases, particularly for complex conditions such as severe asthma, older people and those with multimorbidity chronic obstructive pulmonary disease (COPD), as these conditions tend to have more severe disease and increased comorbidities $[4,5]$. A case illustrating this approach is presented in box 1. Previous articles have described the key treatable traits and their recognition [1,6], and have proposed research methodologies to test the concept [7], but many questions relating to the practical implementation of the treatable traits approach remain and new questions continue to emerge.

In considering our approach to this new treatment paradigm, it may be useful to examine lessons learnt in other disease areas, e.g. cystic fibrosis (CF). Like severe asthma and COPD, CF is both a complex and heterogeneous disease. Yet, in contrast to asthma and COPD, outcomes for CF patients have dramatically improved over the last three decades, mostly due to discoveries and innovations ranging from the identification of the CF transmembrane conductance regulator gene to the implementation of disease-modifying treatments [8]. Importantly, CF was one of the first pulmonary diseases where the importance of specialist multidisciplinary teams was recognised. Patients attending specialist centres achieved superior outcomes than those attending nonspecialised centres [9, 10]. Hence, the provision of multidisciplinary care in CF is now the standard [9]. These major advances in outcomes for patients with CF illustrate nicely what it is possible to achieve when a complex airway disease is managed using multidimensional assessment and targeted therapy, even before treatments that modify the underlying genetic defect are available. We believe that this provides clear support for a similar model in other chronic airway diseases. Specifically, that by combining the recognition of specific treatable traits in each individual and the additional complexities related to older people with multimorbidity, and implementing a multidisciplinary team approach, we may eventually achieve outcome improvements of a similar magnitude to those observed in CF patients. We also urge that this approach is generally applicable to chronic disease management and issue a challenge to other disciplines involved in treating NCDs to consider evaluation of such an approach, e.g. in diabetes and heart failure.

Here, we report the outcomes of the Treatable Traits Down Under International Workshop, held on May 7-8, 2018 in Melbourne, Australia, to critically review the treatable traits approach, identify the importance (hence, priority) of different traits, develop a research agenda to test and validate specific treatable traits approaches, and consider ways to implement this strategy into clinical practice.

\section{Treatable traits: theoretical underpinnings}

What is the treatable traits approach and why is it necessary?

Biology, thus medicine, is complex $[11,12]$. This means that health and disease are emergent properties that result from the interaction of different complex networks at different biological levels (genes, proteins, metabolites, cells, organs and environment) $[11,12]$. Therefore, in order to improve medical diagnosis and treatment it is necessary to gain a better understanding of this biomedical complexity [13-15].

An early approach to address this complexity was based on the concept of "clinical phenotypes", defined as "a single or combination of disease attributes that describe differences between individuals with [the same disease] as they relate to clinically meaningful outcomes" [16]. A strict interpretation of this 
BOX 1 A case of a patient treated according to the treatable traits strategy

\section{Case history}

Denise is 60-year-old female with chronic obstructive pulmonary disease (COPD). She presents to the clinic with cough and exertional breathlessness.

Over the past 12 months she reports experiencing four acute lung attacks, each requiring antibiotics and oral corticosteroids. She is a current smoker with a 44 pack-year history. She reports a history of sinusitis and a fracture of her left wrist 6 years earlier.

Spirometry shows severe airflow limitation with a forced expiratory volume in $1 \mathrm{~s}$ (FEV1) of $1.05 \mathrm{~L}$ (45\% predicted) and a forced vital capacity of $1.90 \mathrm{~L}(66 \%$ predicted), giving a forced expiratory ratio of 0.55 with no bronchodilator response. Chest radiography revealed hyperinflation. Her 6-min walk distance (6MWD) is reduced at $395 \mathrm{~m} \mathrm{(76 \%} \mathrm{predicted).} \mathrm{Her} \mathrm{body} \mathrm{mass} \mathrm{index} \mathrm{(BMI)} \mathrm{is} 22.3 \mathrm{~kg} \cdot \mathrm{m}^{-2}$, her BODE (BMI, airflow obstruction, dyspnoea, exercise capacity) index score is 3 and she reports a modified Medical Research Council dyspnoea scale score of 2 . She expectorates $\sim 50 \mathrm{~mL}$ of whitish/yellow sputum every day.

She is prescribed optimal pharmacotherapy for COPD quadrant D, including budesonide/formoterol 400/12 $\mu \mathrm{g}$ one inhalation twice daily via a dry powder inhaler, tiotropium $18 \mu \mathrm{g}$ daily via a HandiHaler and salbutamol $100 \mu \mathrm{g}$ as required via a pressurised metered-dose inhaler.

When Denise was asked what her biggest problem was related to her chest condition she said, "I have a horrible cough! It's embarrassing. There is lots of phlegm every morning and I have so many flare ups, these limit my activity". Her quality of life score measured by the St George's Respiratory Questionnaire (SGRQ) is impaired with a score of 63.3.

As part of her management Denise underwent a multidimensional assessment guided by the treatable traits model. This revealed a number of treatable traits including:

\begin{tabular}{|c|c|}
\hline Treatable trait & Individualised treatment delivered via case management \\
\hline \multicolumn{2}{|l|}{ Pulmonary domain } \\
\hline Airway eosinophilic inflammation: sputum eosinophils $6.5 \%$ & $\begin{array}{c}\text { Oral corticosteroids (OCSs) } 37.5 \mathrm{mg} \cdot \text { day }^{-1} \text { and sputum monitoring to } \\
\text { guide treatment reduction }\end{array}$ \\
\hline Proneness to lung attacks & $\begin{array}{l}\text { Written action plan, self-management education to identify early signs } \\
\text { of an attack and action, and treatment of the underlying pathology } \\
\text { (airway inflammation and mucus hypersecretions) }\end{array}$ \\
\hline Pathogen colonisation: Haemophilus influenzae & $\begin{array}{c}\text { Antibiotic-based written action plan based on antibiotic sensitive to } \\
\text { pathogen }\end{array}$ \\
\hline \multicolumn{2}{|l|}{ Extrapulmonary domain } \\
\hline $\begin{array}{l}\text { Osteopenia (bone mineral density (BMD) T-score): total body }-1.8 \text {, } \\
\text { hip }-2.4\end{array}$ & Alendronate 70 mg weekly \\
\hline $\begin{array}{l}\text { Sarcopenia lappendicular skeletal muscle mass index } \\
\text { (ASMMI)): } 5.4 \mathrm{~kg} \cdot \mathrm{m}^{-2}\end{array}$ & $\begin{array}{l}\text { Nutritional counselling with a high protein diet and resistance } \\
\text { exercise training }\end{array}$ \\
\hline $\begin{array}{l}\text { Suboptimal self-management skills: inadequate inhaler, } \\
\text { inhaler device polypharmacy and no written action plan } \\
\text { for lung attacks }\end{array}$ & $\begin{array}{l}\text { Self-management education with an antibiotic and OCS-based written } \\
\text { action plan, inhaler device training, and a change of inhaled therapy to } \\
\text { one inhaler platform }\end{array}$ \\
\hline Current smoker, but ready for quit attempts & Smoking cessation counselling and pharmacotherapy \\
\hline
\end{tabular}

The outcomes for this individualised treatable traits approach are outlined as follows:

\begin{tabular}{|c|c|c|c|}
\hline Outcome & Baseline & 3 months & 6 months \\
\hline SGRQ score & 63.3 & $56.3^{\#}$ & $59.2^{\#}$ \\
\hline FEV $1 \%$ pred & 45 & $54^{\#}$ & $50^{\#}$ \\
\hline 6MWD m & 395 & $450^{\#}$ & $488^{\#}$ \\
\hline Mucus volume $\mathrm{mL} \cdot$ day $^{-1}$ & 50 & $25^{\#}$ & $25^{\#}$ \\
\hline $\begin{array}{l}\text { Smoking status cigarettes } \cdot \text { day }^{-1} / \\
\text { exhaled carbon monoxide ppm }\end{array}$ & $20 / 14$ & $0 / 5^{\#}$ & $20 / 12$ \\
\hline BODE index score & 3 & $2^{\#}$ & $2^{\#}$ \\
\hline Self-management skills & Suboptimal & Optimal & Optimal \\
\hline
\end{tabular}

\#: clinically significant response.

The evaluation of treatable traits is feasible in practice with the use of a multidisciplinary team. When the identified traits are treated using a case management approach, improvements are achieved in the three treatable trait domains in addition to overall health status. 
definition may suggest that "clinical phenotypes" are mutually exclusive, whereas we know that diseases often present with several, nonmutually exclusive characteristics. Accordingly, the concept of "clinical phenotypes" evolved to that of "treatable traits", defined as therapeutic targets identified by "phenotype" or "endotype" through validated biomarker(s) [6]. The term "endotype" refers to a "subtype of a disease defined functionally and pathologically by a molecular mechanism or by treatment response" [17]. In contrast, a "biomarker" is "a characteristic [not necessarily a molecule; lung function or chest imaging may work as biomarkers too] that is objectively measured and evaluated as an indicator of normal biological processes, pathogenic processes, or biological responses to a therapeutic intervention" [18]. We refer to these indicators as trait identification markers.

Importantly, potential treatable traits are not restricted to the lungs. It has been recognised since its original proposal that there are pulmonary, extrapulmonary and even behaviour/lifestyle risk factors that deserve specific treatment if present [6]. In essence, it is hoped that the adoption of a strategy based on the recognition and treatment of validated treatable traits may improve the efficacy and safety of therapies of complex human diseases in general, and chronic airway diseases in particular [7, 19, 20]. Needless to say, this hypothesis requires formal, prospective, validation in appropriately designed clinical trials [7]. The first steps to test/validate this approach are to define how to recognise traits and how to assess their relative importance.

\section{How should we identify candidate treatable traits?}

First, it is important to recognise that a given patient may have more than one treatable trait (actually, this is often the case). In other words, treatable traits are not mutually exclusive. Having said this, candidate treatable traits should fulfil three characteristics: 1) clinically relevant, 2) identifiable and measurable, and 3) treatable (table 1).

\section{Clinical relevance}

A trait is required to be clinically important, i.e. a trait should be associated with specific disease outcomes such as symptoms, health status, risk of future events (e.g. exacerbations, cardiovascular events, cancer), lung function decline, prognosis and/or death. In essence, we need to identify treatable traits that matter $[4,7,21]$.

\section{Identifiable and measurable}

A trait identification marker is used to objectively identify the presence of a trait, in preparation for targeted therapy. Typically, this would be a biomarker, such as blood eosinophil count to recognise type 2 airway inflammation. However, it can also be a questionnaire result, such as the Nijmegen Questionnaire score for dysfunctional breathing or an anxiety and/or depression scale to recognise psychological dysfunction. The markers that are used as diagnostic criteria for the trait should have a high specificity, allowing a high degree of confidence in "ruling in" or identifying the presence of the trait. In some situations, this gold standard marker may be prohibitively resource intensive to be measured in all patients with airways disease. For example, at present, the use of high-resolution computed tomography to identify bronchiectasis is not feasible in all patients with chronic airway disease because of cost, the cumulative risk from radiation exposure and the incidental findings that may occur. In these situations, there may be a role for a high-sensitivity screening tool to "rule out" the diagnosis in the majority of patients and select a subset for further investigation. In any case, there is a need to identify and validate new trait identification markers with better operating characteristics, improved feasibly and/or greater cost efficiency.

\section{TABLE 1 Key components of treatable traits and research opportunities}

Domain Essential Clinical application Research opportunity

$\begin{array}{ccc}\text { Clinically relevant } \quad \text { Yes } & \begin{array}{c}\text { Trait predicts/associates with clinically } \\ \text { important outcomes. }\end{array}\end{array}$

Trait identification marker Yes Identifies the presence of a trait. Appreciation of the measurement characteristics of the test (i.e. sensitivity, specificity) is required for optimum use. A test with high specificity is required to "rule in" the presence of a trait. A highly sensitive test can be used to screen, or "rule out" the presence of a trait.

Treatable
Yes
Trait is responsive to a specific targeted therapy. Established via randomised controlled trials.
Identify and/or quantify clinical relevance.

New markers at lower cost or improved feasibility. Novel diagnostics, e.g. artificial intelligence-based probabilities derived from composite molecular signatures. Mechanism-oriented research to yield better molecular diagnostics for more precise identification of subsets.

Discovery science to identify new treatment for "untreatable" traits. Implementation science to define best way to treat the traits in clinical practice. 
Treatable

A trait should be able to be effectively treated in order to be called a "treatable" trait. Ideally, treatability is determined in a randomised controlled trial (RCT) but research opportunities exist in treatment implementation and optimisation in real life, so the effect size is increased and/or side-effects are reduced [22]. Furthermore, even for those traits that are not currently treatable, or are only partly treatable, there are discovery science opportunities to identify new treatments for these traits.

How can we assess the importance of specific treatable traits?

Are some traits more important than others? Should they receive greater priority in patient assessment and management? How should traits be prioritised? The significant resource requirements for assessing all of the potential pulmonary, extrapulmonary and risk factor/behavioural traits, so that targeted and individualised interventions can be implemented, highlights the need to prioritise certain traits above others $[4,7,21]$. An example of this prioritisation would be to target a few traits exclusively, such as symptoms and exacerbations in COPD (currently recommended by the Global Initiative for Chronic Obstructive Lung Disease (GOLD)), and adding eosinophilic inflammation, also now recommended by GOLD (shown previously to be a valid identifying marker of the presence of eosinophilic airway inflammation, a higher exacerbation risk and better treatment response to inhaled corticosteroids) [23-27].

But are there other traits that need to be addressed, and with what priority? Traits could be prioritised based on their clinical impact, i.e. their severity, prevalence or their impact on specific outcomes (exacerbations, symptoms and health status, death, etc.). McDonald et al. [4] assessed clinical impact in an analysis of treatable traits in severe asthma using the Australasian Severe Asthma Web-based Database (SAWD) registry. The authors identified treatable traits that were predictive of future exacerbation risk. From 24 identified traits, 10 were associated with an increased risk of future asthma attacks. The strongest predictors were past-year exacerbation, depression, vocal cord dysfunction, inhaler device polypharmacy and obstructive sleep apnoea (figure 1a) [4]. This list of traits could be a potential "hit list" to begin prioritisation for implementation of the treatable traits approach in severe asthma, and also as a model to allow prioritisation in asthma and COPD (figure 1a). However, we also recognise the need to identify the traits that impact other outcomes, particularly the long-term natural history of a diseased state, or the development of a new disease.

Other ways to prioritise traits include connectivity and patient impact. A trait that is a nodal point in a disease network may influence multiple other traits and therefore would be important to target $[11,13,28]$. For example, in obese COPD patients, targeting treatment to the single trait of obesity led to significant improvements, not only in body weight, but also in several other important traits such as skeletal muscle strength, exercise tolerance, depression, and several cardiovascular and metabolic risk markers (figure 1b) [29].

Patient impact, i.e. a trait rated as a high priority by patients, is another way to prioritise the importance of traits. At present scant data exist to inform this approach in airway diseases. In one study, patients with asthma and COPD aged $\geqslant 55$ years underwent a multidimensional assessment to characterise traits where patients ranked the importance of their individual traits [30]. The most important from the patient's perspective included dyspnoea, activity limitation, airflow limitation, airway inflammation and obesity [30]. Importantly, these rankings were different from those provided by their physicians. However, after including patients in shared decision making, which involved an explanation of the trait, its impact, how it was treated and sharing the rating of the physician, patients became more engaged in the adoption of a complex treatable trait intervention, which led to improved outcomes [31]. These results highlight the importance of seeking patient preferences in prioritising traits and targeted treatments.

\section{Current data and future research needs}

There are limited data currently available on the prevalence of treatable traits in different populations, but data for some traits can be derived from existing observational studies and disease registries. With this in mind, the prevalence of traits such as eosinophilic airway disease and airflow limitation have been well described in asthma and COPD settings, whereas the prevalence of other traits such as vocal cord dysfunction have predominantly been reported in the setting of severe asthma [32, 33] and not in unselected airway diseases or patients with COPD. The reported prevalence of a trait is likely to vary according to the threshold used, diagnostic label, severity and setting in which it is described. This variability is clearly illustrated by the trait of eosinophilic airway inflammation: estimates from the Severe Asthma Research Programme, TENOR II cohorts and the UK primary care database indicate that, depending on asthma severity, the prevalence of blood eosinophils $\left(\geqslant 0.3 \times 10^{9} \mathrm{~L}^{-1}\right)$ in asthma patients ranges between 26\% and 43\% [34-36]. By contrast, in the Australasian SAWD, eosinophilic airway inflammation (defined using a composite of sputum eosinophilia $>3 \%$, exhaled nitric oxide fraction $\geqslant 30 \mathrm{ppb}$ or blood eosinophils $\geqslant 0.3 \times 10^{9} \mathrm{~L}^{-1}$ ) was present in $53 \%$ of patients with severe asthma and $60 \%$ 


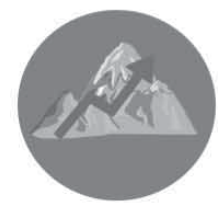

Exacerbation prone $2.07(1.66-2.58)$

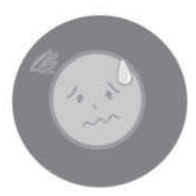

Anxiety

$1.27(1.03-1.56)$

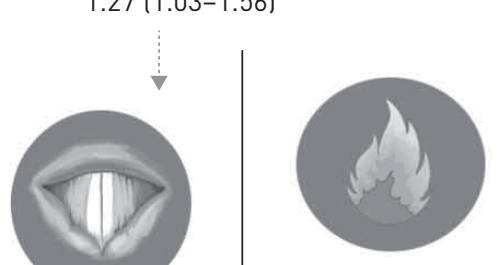

Systemic

Vocal cord dysfunction $1.51(1.22-1.88)$
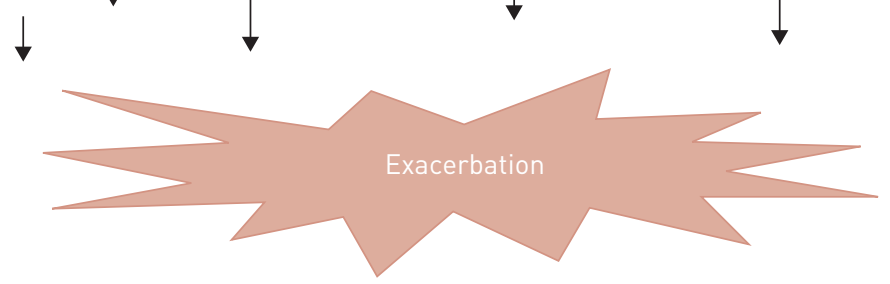

b)

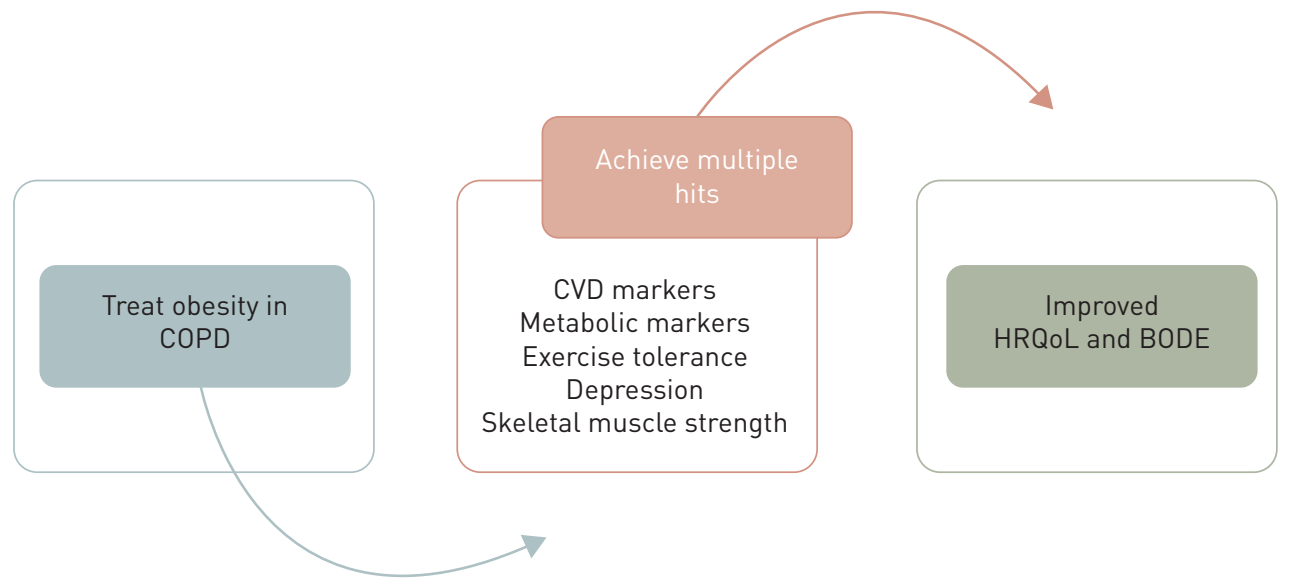

FIGURE 1 a) Treatable traits significantly $(p<0.05)$ associated with increased risk of exacerbations over time. Numbers indicate the incidence rate ratio $(95 \% \mathrm{Cl})$ for the total effect of each trait on exacerbations over time. Adapted with permission from [4]. Illustrations by Olivia J. McDonald. b) An example of an intervention targeting one trait but achieving multiple positive outcomes, including health-related quality of life (HRQoL) and BODE (body mass index, airflow obstruction, dyspnoea and exercise capacityl index [29]. COPD: chronic obstructive pulmonary disease; CVD: cardiovascular disease.

of patients with nonsevere asthma [4]. A further complication arises when comparing asthma and COPD, as the majority of COPD studies used a blood eosinophil cut-off of $\geqslant 2 \%$ of total white cell count at baseline, rather than an absolute cut-off. For instance, in the ECLIPSE study, 84\% of participants with COPD had blood eosinophils $\geqslant 2 \%$ on at least one measurement (equivalent to a $\geqslant 0.15 \times 10^{9} \mathrm{~L}^{-1}$ cut-off for the majority of participants) [37]. However, trait stability over time must also be considered as only $37 \%$ of patients with COPD had blood eosinophils persistently $\geqslant 2 \%$ of total white cell count over a 3 -year period, but another $49 \%$ met this criterion intermittently [27].

The treatable traits approach allows for rapid management shifts in response to dynamic changes in trait identification markers, which diverges from traditional thinking of relatively fixed diagnoses. The normalisation or absence of a trait identification marker may indicate successful management, which may instigate clinical review of management strategies. Dimensional approaches that consider the severity, rather than simply presence, of traits may also assist in guiding management of traits that vary over time. 
TABLE 2 Comparison of candidate treatable traits in the literature

Agusti et al. 2016 [6]

GIBSon et al. 2010 [5]

PAvord et al. 2017 [1]

\section{Pulmonary treatable traits}

Airflow limitation/obstruction

Exercise intolerance

ASM contraction

Loss of elastic recoil (emphysema)

Airway mucosal oedema

\section{Eosinophilic airway inflammation}

Mixed airway inflammation

Chronic bronchitis

Infection

Frequent chest infection

Airway bacterial colonisation

Pathogen colonisation

Bronchiectasis

Cough reflex hypersensitivity

Pre-capillary pulmonary hypertension

Mucus hypersecretion

Chronic respiratory failure

Oxygen desaturation

Arterial hypoxaemia

Arterial hypercapnia

Extrapulmonary treatable

traits

Deconditioning
$\mathrm{FEV}_{1} / \mathrm{FVC}<0.7$ (or LLN)

$x$

Bronchodilator reversibility, peak expiratory flow variability, positive $\mathrm{PC}_{20}$

Chest CT, DLCo, compliance

Chest CT, spirometry-induced bronchoconstriction

Sputum eosinophils, blood eosinophils, FenO, (periostin)

X

Cough and sputum 3 months for 2 years (no eosinophilic airway inflammation]

$$
X
$$

X

Sputum culture, quantitative PCR

\section{$x$}

\section{Chest CT}

Capsaicin challenge, cough counts, cough questionnaire

Doppler echocardiography, $\mathrm{BNP}, \mathrm{RHC}$

$$
\mathrm{X}
$$

$$
\begin{aligned}
& P_{\mathrm{aO}_{2}}<55 \mathrm{mmHg} \\
& P_{\mathrm{aCO}_{2}}>45 \mathrm{mmHg}
\end{aligned}
$$

CPET, 6MWD
$\mathrm{FEV}_{1} / \mathrm{FVC}<70 \%$ and $\mathrm{FEV}_{1}<80 \%$ predicted or use agreed standards 6MWD (distance <350 m)

$$
\mathrm{X}
$$

$X$

$x$

$$
\text { Sputum eosinophils }>3 \%
$$

Sputum neutrophils $>61 \%$; paucigranulocytic if neutrophils $<61 \%$ and eosinophils $<3 \%$, mixed if neutrophils $>61 \%$ and eosinophils $>3 \%$

$\mathrm{X}$ $x$

$\geqslant 2$ antibiotic courses in 12 months $\mathrm{X}$

Sputum culture, presence of a recognised bacterial pathogen

$x$

$x$

$x$

$\geqslant 25 \mathrm{~mL}$ of mucus produced daily for the past week in the absence of an infection

$\mathrm{SpO}_{2}<90 \%$ either at rest or during 6MWD test

\section{$x$}

$x$

$x$
FEV $1 / F V C<L L N$ bronchodilator reversibility and short-term PEF variability consistent with variable airflow obstruction and large component of ASM contraction, ICS/ OCS response consistent with inflammation associated with airflow limitation (i.e. mucosal oedema, mucus plugging), loss of airway support probable if imaging or physiological evidence of emphysema; more work is needed to identify tests capable of discriminating these processes

FeNO, serum periostin, blood eosinophils, sputum eosinophils

$X$

$X$

Sputum culture, sputum PCR

$x$

$x$

$x$

$X$

Increased cough reflex sensitivity li.e. capsaicin), increased cough counts, cough symptom scores

$X$

$x$

$x$

$x$

$\mathrm{X}$

$X$ 
TABLE 2 Continued

Agusti et al. 2016 [6]

GIBSon et al. 2010 [5]

Pavord et al. 2017 [1]

Activity limitation

Obesity

Overweight

Obesity/deconditioning

Cachexia

Malnutrition

Obstructive sleep apnoea syndrome

Cardiovascular disease

Cardiac dysfunction

Gastro-oesophageal reflux disease

Upper airway diseases

Rhinosinusitis

Inducible laryngeal obstruction (VCD)

Psychiatric disorders

Depression

Anxiety/other behavioural aspects, including breathing pattern disorders or VCD

Persistent systemic inflammation

Treatment-associated morbidity

Other pulmonary or nonpulmonary condition

Anaemia

Dysfunctional breathing

Behavioural treatable traits Smoking and other environmental exposures
X

$\mathrm{BMI}$

X

$x$

$\mathrm{BMI}$

$x$

Questionnaires, polysomnography

ECG, Doppler echocardiography, BNP

$x$

Gastrointestinal endoscopy, $\mathrm{pH}$ monitoring

History and examination, imaging

Fibreoptic laryngoscopy, flow-volume curve, dynamic neck CT

Questionnaires, psychologist/liaison psychiatrist assessment

Questionnaires, psychologist/liaison, psychiatrist assessment

High-sensitivity CRP

$x$

$x$

$x$

$x$

Cotinine, eCO
Self-report, defined as self-reported impairment because of an inability to achieve personal activity goals

$$
\mathrm{BMI}>30 \mathrm{~kg} \cdot \mathrm{m}^{-2}
$$$$
\text { BMI } 25-30 \mathrm{~kg} \cdot \mathrm{m}^{-2}
$$

$x$

$\mathrm{BMI}<20 \mathrm{~kg} \cdot \mathrm{m}^{-2}$

$\mathrm{X}$

$x$

Chest radiography, echocardiogram, NT-proBNP >1000 fmol. $\mathrm{mL}^{-1}$

$x$ $x$

$x$

HADS depression domain score $\geqslant 8$, GADS score $>5$ suggests depression

HADS anxiety domain score $\geqslant 8$

High-sensitivity CRP $>3 \mathrm{mg} \cdot \mathrm{L}^{-1}$

$x$

$x$

Haemoglobin $<120 \mathrm{~g} \cdot \mathrm{L}^{-1}$ for females

$$
\text { or }<140 \mathrm{~g} \cdot \mathrm{L}^{-1} \text { for males }
$$

Nijmegen Questionnaire Total score $\geqslant 23$

Self-report and eCO, admit to smoking and eCO $\geqslant 10$ ppm or deny smoking and show eCO $x$

$x$

$X$

$\mathrm{BMI}$, cardiorespiratory exercise test

$x$

$x$

$x$

Oesophageal manometry

Suggestive symptoms, imaging

$x$

HADS

Disproportionate breathlessness, air hunger, frequent sighs, dizziness, light headed, tingling hands and face, chest tightness, increased Nijmegen Questionnaire score, noisy inspiration

$x$

ACE inhibitor-associated cough, breathlessness or tiredness secondary to $\beta$-blocker

Focal chest signs, prominent crackles, clubbing, weight loss, haemoptysis, chest pain, cardiac history or risk factors, restrictive spirometry, abnormal chest radiograph or CT

$x$

$x$

Smoking history, urinary cotinine, eCO Continued 
TABLE 2 Continued

$x$

Exacerbation management

Nonadherence

Inhaler device polypharmacy

Inhaler device technique

Social and behavioural issues

Exposure to sensitiser (allergen, occupational)

Symptom perception

Side-effects of other treatments

Family and social support

\section{$X$}

Agusti et al. 2016 [6]

GIBSON et al. 2010 [5]

Self-report, patient does not possess

written action plan or does not use the prescribed plan during exacerbations

Prescription refill rate, chipped inhalers

$\geqslant 3$ different types of inhaler devices being used

Observation, training devices

RASTs, SPTs

Mismatch between

subjective and objective findings

Monitored withdrawal

None given
Self-report by a series of open-ended questions, reported use of $<80 \%$ of prescribed treatment

Medication review, prescription of $\geqslant 3$ different inhaler devices

Direct observation and standardised assessment, technique rated as inadequate

$X$

$\mathrm{X}$

X

$x$
Pavord et al. 2017 [1]
Prescription refill rates

$X$

Drug concentrations, FeNO suppression test, chipped inhalers

Social history, home visit, school and workplace information

Atopic tendency (presence of disease, family history), history (i.e. latency), relevant exposures, SPTs, RASTs

$X$

$X$

FEV1: forced expiratory volume in $1 \mathrm{~s}$; FVC: forced vital capacity; LLN: lower limit of normal; PEF: peak expiratory flow; ASM: airway smooth muscle; ICS: inhaled corticosteroid; OCS: oral corticosteroid; 6MWD: 6-min walk distance; PC20: provocative concentration causing a $20 \%$ fall in FEV1; CT: computed tomography; DLCO: diffusing capacity of the lung for carbon monoxide; FeNO: exhaled nitric oxide fraction; BNP: brain natriuretic peptide; $\mathrm{RHC}$ : right heart catheterisation; $\mathrm{SpO}_{2}$ : arterial oxygen saturation measured by pulse oximetry; $P_{\mathrm{aO}} \mathrm{O}_{2}$ : arterial oxygen tension; $\mathrm{PaCO}_{2}$ : arterial carbon dioxide tension; CPET: cardiopulmonary exercise testing; BMI: body mass index; NT-proBNP: N-terminal pro-brain natriuretic peptide; VCD: vocal cord dysfunction; HADS: Hospital Anxiety and Depression Scale; GADS: Goldberg Anxiety and Depression Scale; CRP: C-reactive protein; ACE: angiotensin-converting enzyme; eCO: exhaled carbon monoxide; RAST: radioallergosorbent test; SPT: skin prick test. " $\mathrm{X}$ " indicates that the trait was not included in the publication.

Table 2 illustrates reviews of treatable traits, and similar approaches show considerable variation in both traits and trait definitions proposed to date $[1,5,6]$. Consensus on trait identification markers can be agreed through a Delphi process and this will provide a bedrock on which an evidence base can be established. A key consideration will be the composition of the expert panel contributing to the Delphi process, ensuring inclusion of a range of healthcare professionals and specialist interests.

Thus, given these limitations of existing evidence, a specific research programme is required to evolve the treatable trait strategy from a useful conceptual tool to an evidence-based approach to diagnosis and treatment. Table 3 lays out some of the key questions that need to be answered and suggests appropriate methodologies to address them. The immediate priority must be to avoid replicating the challenges previously encountered with both COPD and asthma, particularly the overlap of these conditions, where differences in definition result in conflicting estimates of the prevalence of the conditions [38] and hampered interpretation of the evidence [39]. Once trait identification markers are agreed, existing datasets from large observational cohorts can be used to estimate the prevalence of individual treatable traits in different populations. Severe asthma registries, COPD observational cohorts and cohorts derived from random population samples will provide complementary information on trait prevalence, variation in prevalence according to diagnosis of asthma or COPD, and association with current symptoms, disease control and health status. Longitudinal cohorts will be required to determine trait stability over time and associations with future risk, particularly in relation to exacerbations and lung function decline.

Candidate traits that are common and found to be strongly associated with the domains of symptom control, health status or risk of future events should represent priorities for investigation and treatment. 
TABLE 3 Key treatable traits research questions

Question

How should we define individual traits?

What is the prevalence of the different traits?

Which treatable traits matter?

Is a broad or narrow/focused approach to managing treatable traits preferable?

\author{
What is the best treatment for specific \\ traits?
}

How best to implement treatable traits?

\section{Methodology}

Delphi process/consensus statement

Existing datasets, supplemented by prospective data collection where necessary

Existing cross-sectional and longitudinal datasets for quality of life, control and future risk

Prospective randomised controlled trial, three arms with stratification by baseline diagnosis asthma/chronic obstructive pulmonary disease: guideline based; broad approach at baseline; focused approach at baseline

Master protocol randomised controlled trials

Implementation studies: dynamic control panel embedded in electronic record

Some traits may require considerable resources for their investigation and others may be at present suboptimally or ineffectively treated. Current inability to treat a trait should not be an absolute barrier to its recognition, as traits that are strongly associated with important outcomes but are not yet treatable may represent important avenues for development of new therapies. Consumer involvement should also be used to inform the setting of research priorities. It is clear that patients and healthcare professionals differ over concepts such as what constitutes acceptable asthma control [40] and the priority for treatment of specific traits [30], and similar differences may exist over the relative importance of traits which affect current symptoms versus future risk.

When testing the utility of the treatable trait strategy through RCTs, key considerations include the disease population in which the strategy is tested and which traits are targeted. The decision should be made whether to test a treatable trait intervention within a population of patients with the disease label of either asthma or COPD, or to include participants with symptoms of airway diseases irrespective of the current label. If trait prevalence studies suggest that the label of asthma or COPD is associated with particular sets of traits, then study designs should reflect this and may differ. For example, a treatable traits study in the setting of asthma may prioritise different traits from a study in the setting of pulmonary rehabilitation for COPD. The diagnosis of asthma or COPD should not be the end of the diagnostic procedure; it should be an intermediate step [20]. Linked to this is the question of whether to assess all traits at once, the "broad" approach akin to systematic assessment in the severe asthma clinic [41], or to target the most prevalent or most important traits, the "focused" approach. An example of a focused approach that has been proposed would be to target the two traits of eosinophilic airway inflammation and airflow limitation, adjusting the use of inhaled corticosteroids and bronchodilators accordingly [42]. Powell et al. [24] used this approach in the antenatal clinic setting for managing asthma in pregnancy and established that it was superior to a step-based approach, and GreEN et al. [26] reported similar findings in patients with severe asthma. The focused approach may lend itself more to implementation in primary care, where targeting eosinophilic airway inflammation and airflow limitation can be achieved effectively in the majority [6]. A broad approach requires a more time-consuming systematic assessment, but the relative simplicity of the focused approach may limit the potential benefit of the interventions. A resource-efficient design may be a "phased" approach, where patients are initially treated according to a focused algorithm but progress to an extended or "broad" assessment if they do not achieve acceptable disease control.

Conventional parallel-group RCTs are the most appropriate model for testing the efficacy of a treatable traits-based multicomponent intervention. However, they are not the most efficient way to determine the best methods of assessment and treatment for individual traits. Given the many possible traits, and the heterogeneity of the populations, a programme of trials providing evidence for the investigation and management of each trait would take several decades to complete. A more feasible methodology may be to design master protocols, to simultaneously assess interventions across multiple traits [43]. An adaptive platform RCT of treatable traits could also aid implementation of the research findings, as once a treatment is shown to be superior all new participants are allocated to this arm [44]. Implementation may be further assisted by a treatable traits dynamic control panel embedded within the electronic health record [45]. Such a system (figure 2) could draw on existing data in the health record, such as blood eosinophil levels and spirometry, and highlight priority traits to target in an individual. This sort of 


\begin{tabular}{lll}
\hline Domain & $\begin{array}{c}\text { Individualised } \\
\text { risk }\end{array}$ & Traits in this individual \\
\hline Quality of life & VCD I Rhinosinusitis I Eosinophilic airway inflammation I Reflux \\
\hline $\begin{array}{l}\text { Airway disease control } \\
\text { Future risk }\end{array}$ & VCD I Eosinophilic airway inflammation \\
\hline
\end{tabular}

FIGURE 2 Example of a dynamic control panel that could be embedded in an electronic patient record. VCD: vocal cord dysfunction; CVD: cardiovascular disease. Traits will appear based on the presence of relevant medication, diagnosis coding or test results. Colours in the individualised risk column reflect the composite risk score for the individual based on identified traits. Priority traits for treatment are highlighted in green based on their association with risk and treatment responsiveness.

algorithmically assisted medicine may facilitate a treatable traits approach in time- and resource-limited settings, and should be a focus for implementation research.

\section{Study outcomes for research: concept of burden}

Assessing the impact of a new strategy for managing airway diseases requires some consensus about the end-points that are appropriate for assessing that impact. The concept of "burden of disease" has been used as a common currency for describing the impact of diseases and risk factors. Burden of disease represents a measure of health loss and is estimated in two, mutually exclusive, components: years of life lost due to premature death and years of life lived with disability. The former is the sum of life expectancies, at the time of death, of all people whose death is attributed to the specified disease or risk factor. The latter is the sum of all periods of disability, weighted by the level of disability, attributed to the specified disease or risk factor. These two components are then summed to give the burden of disease, attributed to the specified disease or risk factor, in units of disability-adjusted life years [46]. This approach has been widely used to estimate and analyse the global burden of disease.

A strength of this approach is its generalisability across diseases and global contexts. The holistic approach to assessment of impact is a further strength. One potential limitation of this approach is that descriptors used to assign levels of disability to various stages of disease (mild, moderate and severe) are largely based on regular symptoms and limitations [47]. This does not account for the impact of exacerbations, which are sporadic in nature and are a major component of the impact of the disease that the treatable traits strategy seeks to mitigate. These limitations, particularly the latter, can and should be addressed in future work on the burden of disease measures. Another limitation may be that these are population estimates and, in an era of precision, individualised, medicine there may be substantial interindividual variability [48].

\section{How can we implement the treatable traits approach into practice?}

Assuming that appropriate research shows that the treatable traits approach is more efficient, effective and safer than the traditional approach, implementing it in clinical practice is a major challenge. Yet, there are a number of potential ways that could be considered here, such as using guidelines, pulmonary rehabilitation, and/or multidisciplinary team assessment and treatments.

\section{Guidelines as a vehicle to implement a treatable traits strategy}

Guidelines present a comprehensive approach to airway disease management and in doing so they may be already dealing with many of the issues identified in the treatable traits approach. However, there are important differences between the treatable traits strategy discussed earlier and the current guidelines approach. So far, guidelines do not advocate the use of biomarkers to select therapy, although based on current evidence this may change in the near future [27]. In general, guidelines tend to be based on "lumping", with the level of treatment determined by the degree of symptoms and risk of future events. In contrast, the treatable traits approach personalises treatment based on "splitting", with systematic identification and treatment of the disease characteristics that are evident in each individual and that contribute to poor respiratory health. The consequence of the "lumping" approach is that there may be, for example, untreated eosinophilic inflammation when symptoms alone are used to guide therapy. Eosinophilic inflammation often occurs in patients with COPD but can also occur in those with mild asthma, as well as poor symptom perceivers, where there is a weak association between eosinophilic inflammation and symptoms [49]. Several RCTs now show that biomarker-guided therapy directed at the treatable trait of type 2 airway inflammation is superior to symptom-guided therapy [24, 26, 50]. Moreover, how to efficiently implement this approach in primary or secondary healthcare settings remains unclear [6]. Finally, even though some guidelines, e.g. the New Zealand asthma guidelines [51], already recommend the novel approach of assessing treatable traits in a systematic manner within four domains 
(overlapping disorders, comorbidities, environmental and lifestyle factors) at each consultation, and before using the stepwise approach to asthma drug treatment, the successful implementation of existing guidelines remains a challenge $[52,53]$.

Pulmonary rehabilitation as a vehicle to implement a treatable trait strategy

Pulmonary rehabilitation, defined as the delivery of "patient-tailored therapies ... designed to improve the physical and psychological condition of people with chronic respiratory disease and to promote the long-term adherence to health-enhancing behaviors" is a highly effective intervention to improve wellbeing in people with chronic respiratory disease [54]. Although pulmonary treatable traits are infrequently addressed directly in pulmonary rehabilitation, many important extrapulmonary and behavioural/lifestyle traits are addressed routinely. Examples include the extrapulmonary treatable trait of deconditioning, addressed through provision of an individualised exercise training programme, and behavioural treatable traits related to inhalation technique, adherence to treatment, smoking cessation, and family and social support.

Pulmonary rehabilitation is a complex, multicomponent intervention that can be considered as a "stacked" approach to management [7]. This provides the opportunity to address multiple treatable traits at once, which may be of particular advantage in people with more severe airway disease. Such an approach requires patients to make health behaviour changes across multiple domains. Pulmonary rehabilitation programmes provide an environment where this can be supported by case management and coordination of care. Another advantage of embedding the treatable traits approach in pulmonary rehabilitation is that measurement of patient-centred outcomes (e.g. health status) is strongly embedded in routine rehabilitation practice.

While pulmonary rehabilitation offers opportunities for implementation of the treatable traits approach, there are some potential limitations that need to be considered. The "stacked" nature of pulmonary rehabilitation could be one by limiting our understanding of the mechanisms of treatment response. For example, there is strong evidence of improvement in the extrapulmonary treatable traits of anxiety and depression following pulmonary rehabilitation, but it is not clear which components of rehabilitation confer these benefits [55]. Another potential limitation is that the treatable traits approach should span the spectrum of airway diseases, but the benefits of pulmonary rehabilitation for those with mild disease have not been convincingly demonstrated [56, 57]. Finally, access and uptake of pulmonary rehabilitation remain suboptimal across the world [58], which may limit its utility as a vehicle to deliver the treatable traits approach.

\section{Multidisciplinary teams as a vehicle to implement a treatable traits strategy}

Evidence suggests that optimal management of patients with chronic airway diseases requires a multidimensional assessment and targeted treatments [31, 54, 59, 60]. Engaging a multidisciplinary team enables the management of the complexities that characterise this patient population, and could be a useful platform to identify treatable traits and to implement a targeted treatment programme based on these traits. A multidisciplinary team approach for management of severe asthma and COPD has been shown to reduce hospital admissions, improve health status, reduce exacerbations and reduce the number of bed days [61-63].

The minimum recommended personnel for the multidisciplinary team within the severe asthma clinic include a respiratory physician/pulmonologist, a specialist nurse and a pulmonary function scientist, but a speech pathologist, dietician, psychologist and physiotherapist are also necessary to address the complexities associated with the disease [59]. Referral pathways to other specialities for the treatment of common comorbidities or traits (e.g. gastro-oesophageal reflux disease, sinusitis and cardiovascular disease) should also form part of a multidisciplinary team protocol. In COPD the team is very similar. Using a multidisciplinary team meeting format, patients can be presented by the case manager to discuss the individual's identified treatable traits using a multidimensional assessment approach, then treatment of the identified traits can be planned and treatment pathways actioned [5, 31, 64].

While there is good evidence for the benefits of the multidisciplinary team approach in managing airway diseases, there are no published RCTs that have utilised the multidisciplinary team in the evaluation of the treatable traits approach. This identifies another important area for future research.

\section{Knowledge translation}

A further important consideration for the implementation of the treatable traits strategy is knowledge translation. We must consider ways of bringing about behaviour change of multidisciplinary clinicians to successfully create a paradigm shift in disease management. In an era of social media, artificial intelligence and information technology these strategies need to be embraced to launch public health campaigns 
promoting the approach and to provide training for clinicians. These strategies could be supported by our international respiratory communities such as the European Respiratory Society and perhaps even the World Health Organization.

\section{Expansion and future development of treatable traits: who, where and what?}

Treatable traits are proposed as a general framework to use when managing any patient with airway diseases. Yet, to date, much of the research focus has been on people with specific diagnoses of COPD or asthma. Specifying the population in whom to apply the treatable traits approach has implications for which traits are accepted into the framework [20]. For instance, bronchiectasis has been listed as a trait [6], yet can equally be considered an airway disease defined by traits of mucus hypersecretion, dyspnoea, chronic infection and cough [19]. Clear trait identification markers may resolve confusion and open the treatable traits approach to more patients.

How to implement treatable traits across settings also requires consideration. As discussed earlier, guidelines, pulmonary rehabilitation and multidisciplinary team management all show potential as vehicles to implement the treatable traits approach, but good evidence of effectiveness is required. A good test case for treatable traits may be patients hospitalised for an exacerbation of airway disease (asthma or COPD), who often have underrecognised extrapulmonary traits [65]. Here, patients can be intensively investigated, and diagnostic equipment, multiple treatment options and multidisciplinary expertise are often available. Prevention of rehospitalisation is a logical treatment end-point. The corollary, however, is that this is a time where it is proven to be more difficult to engage patients in some interventions, such as pulmonary rehabilitation. Furthermore, while the treatable traits approach may be feasible in well-resourced hospitals, how it may be adapted for settings with limited resources is an open question. Primary care, low- to middle-income countries, rural and remote locations, and other underresourced settings may require a leaner model and a structured approach to facilitate uptake. Research to establish whether traits have similar prevalence and impact across settings, and describe implementation barriers and facilitators, will help develop a translatable and equitable treatable traits approach.

Finally, an expanding evidence base produces an expanding catalogue of potential treatable traits. The scope of the catalogue partly depends on two questions. 1) Do traits need to have a mechanism of effect on the airways or can they include downstream effects of airway diseases and its treatment, as in the case of osteoporosis? 2) Is current treatability necessary? Traits that are potentially, but not yet, treatable may also be considered if they have substantial impact on patient outcomes. For instance, although we have no established treatments specifically targeting neutrophilic airway inflammation, it is identifiable in sputum, prevalent across airway diseases and clinically relevant [66]. Although it is tempting to propose a laundry list of traits, we need first to revise traits to reduce redundancy and carefully consider new traits to ensure an economical and nonburdensome approach. Candidate new traits that may be important for clinical outcomes include skeletal muscle dysfunction, fatigue, osteoporosis, chronic cough, poor diet quality, physical inactivity, frailty, poor disease mastery and poor health literacy.

In terms of potential limitations of the treatable traits strategy, we recognise that at present there are no RCTs that report efficacy or effectiveness data. This is a major limitation and highlights the need for a framework to advance our knowledge in this field, which we offer. One criticism of the approach is the complexity involved in the assessment of many traits within three different domains. This requires additional resourcing and multidisciplinary teams working effectively together. Of course, in addition to efficacy data, health economic evaluation is also a priority, as the strategy is likely to be more resource intensive initially but become cost neutral or cost saving.

\section{Conclusions}

The 2018 Treatable Traits Down Under International Workshop brought together international experts in COPD and asthma, who had an interest in progressing precision medicine in these diseases, using the new paradigm of treatable traits. The meeting provided opportunities to identify and design a research programme that will generate new knowledge in relation to defining consensus of candidate traits, establishing efficacy of the approach and identifying pathways to enable implementation of treatable traits into practice.

Acknowledgements: We would like to acknowledge all of the participants of the Treatable Traits Down Under International Workshop. Illustrations in figure 1a were drawn by Olivia J. McDonald (University of Newcastle, Newcastle, Australia). We thank Steven Maltby and Kim Jones (University of Newcastle) for their support.

Treatable Traits Down Under International Workshop participants: Alvar Agusti, Gary Anderson, Philip P. Bardin, Richard Beasley, Allen Cheng, Li Ping Chung, Vanessa L. Clark, Eli Dabscheck, Shyamali Dharmage, James Fingleton, Peter Gibson, Jo Hardy, Mark Hew, Sarah A. Hiles, Anne E. Holland, Alan James, Krystle Lam, Lata Jayaram, Connie 
Katelaris, Paul Leong, Martin MacDonald, Niamh Mangan, Guy B. Marks, Christine McDonald, Vanessa M. McDonald, Lucy Morgan, Christian Osadnik, Ian D. Pavord, Maximilian Plank, Mary Roberts, Andrew Searles, Hayley See, Aislin Spencer, John Upham and Peter A.B. Wark.

Conflict of interest: V.M. McDonald reports grants from GlaxoSmithKline during the conduct of the meeting; and grants and speaker fees from GlaxoSmithKline and AstraZeneca, personal fees for meeting organisation from Menarini, and grants from Cyclopharm Ltd, outside the submitted work. J. Fingleton reports travel to the educational event "Treatable Traits Down Under", at which the ideas contained within this paper were developed, from GlaxoSmithKline; speaker fees for educational events and support to attend educational events from AstraZeneca Ltd and Boehringer Ingelheim, and grants from GlaxoSmithKline and Fisher \& Paykel Ltd, New Zealand, outside the submitted work. A. Agusti reports grants and personal fees from AstraZeneca and Menarini, and personal fees from Chiesi, GlaxoSmithKline, Nuvaira and Boehringer Ingelheim, outside the submitted work. S.A. Hiles' salary was supported by a grant from GlaxoSmithKline, paid to her institution (University of Newcastle), during the conduct of the meeting. S.A. Hiles also reports salary from a grant from AstraZeneca, paid to her institution, outside the submitted work. V.L. Clark receives a fellowship from the National Health and Medical Research Council Centre of Research Excellence in Severe Asthma. A.E. Holland has nothing to disclose. G.B. Marks reports grants from GlaxoSmithKline (Australia) and from AstraZeneca (Australia), outside the submitted work. P.P. Bardin reports personal fees from GlaxoSmithKline, Boehringer Ingelheim and AstraZeneca, during the conduct of the study. R. Beasley reports grants from the Health Research Council of New Zealand, grants and personal fees from AstraZeneca and GlaxoSmithKline, and grants from Genentech, outside the submitted work. I.D. Pavord reports personal fees from AstraZeneca, GlaxoSmithKline, Boehringer Ingelheim, Sanofi/Regeneron, Circassia, Merck, Novartis, Mundipharma, Teva, Knopp and Roche/ Genentech, and grants and personal fees from Afferent and Chiesi, outside the submitted work. P.A.B. Wark receives grants from The University of Newcastle, John Hunter Hospital Charitable Trust, grants from Hunter Medical Research Institute, grants from National Health and Medical Research Council, during the conduct of the study; he has taken part in advisory boards for AstraZeneca, Boehringer Ingelheim, Novartis and Vertex, and has received personal fees from AstraZeneca, GlaxoSmithKline and Vertex, outside the submitted work. P.G. Gibson reports grants and personal fees from AstraZeneca, GlaxoSmithKline and Novartis, and grants from NHMRC (Australian Government), outside the submitted work.

Support statement: The meeting was gratefully supported by GlaxoSmithKline (Australia). Funding information for this article has been deposited with the Crossref Funder Registry.

\section{References}

Pavord ID, Beasley R, Agusti A, et al. After asthma: redefining airways diseases. Lancet 2017; 391: 350-400

2 Ebmeier S, Thayabaran D, Braithwaite I, et al. Trends in international asthma mortality: analysis of data from the WHO Mortality Database from 46 countries (1993-2012). Lancet 2017; 390: 935-945.

3 Flood-Page P, Swenson C, Faiferman I, et al. A study to evaluate safety and efficacy of mepolizumab in patients with moderate persistent asthma. Am J Respir Crit Care Med 2007; 176: 1062-1071.

4 McDonald VM, Hiles SA, Godbout K, et al. Treatable traits can be identified in a severe asthma registry and predict future exacerbations. Respirology 2019; 24: 37-47.

5 Gibson PG, McDonald VM, Marks GB. Asthma in the older adult. Lancet 2010; 374: 803-813.

6 Agusti A, Bel E, Thomas M, et al. Treatable traits: toward precision medicine of chronic airway diseases. Eur Respir J 2016; 47: 410-419.

7 Agusti A, Bafadhel M, Beasley R, et al. Precision medicine in airway diseases: moving to clinical practice. Eur Respir J 2017; 50: 1701655.

8 Gibson PG, Peters MJ, Wainwright CE. Targeted therapy for chronic respiratory disease: a new paradigm. Med J Aust 2017; 206: 136-140.

9 Jones AM. Adults with cystic fibrosis should be treated at a specialist centre. Paediatr Respir Rev 2013; 14: Suppl. $1,13-15$.

10 Mahadeva R, Webb K, Westerbeek RC, et al. Clinical outcome in relation to care in centres specialising in cystic fibrosis: cross sectional study. BMJ 1998; 316: 1771-1775.

11 Barabasi AL, Gulbahce N, Loscalzo J. Network medicine: a network-based approach to human disease. Nat Rev Genet 2011; 12: 56-68.

12 Topol EJ. Individualized medicine from prewomb to tomb. Cell 2014; 157: 241-253.

13 Agusti A, Sobradillo P, Celli B. Addressing the complexity of chronic obstructive pulmonary disease: from phenotypes and biomarkers to scale-free networks, systems biology, and P4 medicine. Am J Respir Crit Care Med 2011; 183: 1129-1137.

14 Diez D, Agusti A, Wheelock CE. Network analysis in the investigation of chronic respiratory diseases: from basics to application. Am J Respir Crit Care Med 2014; 190: 981-988.

15 Agusti A, Anto JM, Auffray C, et al. Personalized respiratory medicine: exploring the horizon, addressing the issues. Am J Respir Crit Care Med 2015; 191: 391-401.

16 Han MK, Agusti A, Calverley PM, et al. Chronic obstructive pulmonary disease phenotypes: the future of COPD. Am J Respir Crit Care Med 2010; 182: 598-604.

17 Anderson GP. Endotyping asthma: new insights into key pathogenic mechanisms in a complex, heterogeneous disease. Lancet 2008; 372: 1107-1119.

18 Jones PW, Agusti AGN. Outcomes and markers in the assessment of chronic obstructive pulmonary disease. Eur Respir J 2006; 27: 822-832.

19 Boaventura R, Sibila O, Agusti A, et al. Treatable traits in bronchiectasis. Eur Respir J 2018; 52: 1801269.

20 Agusti A, Pavord ID. Do we really need a new classification of airway diseases? Lancet Respir Med 2018; 6: 891-893.

21 Baggott C, Beasley R. Triaging treatable traits in asthma. Respirology 2019; 24: 5-6.

22 Roche N, Reddel HK, Agusti A, et al. Integrating real-life studies in the global therapeutic research framework. Lancet Respir Med 2013; 1: e29-e30. 
23 Global Initiative for Chronic Obstructive Lung Disease. Global Strategy for the Diagnosis, Management, and Prevention of Chronic Obstructive Pulmonary Disease. 2019. https://goldcopd.org/wp-content/uploads/2018/11/ GOLD-2019-v1.7-FINAL-14Nov2018-WMS.pdf Date last accessed: February 22, 2019.

24 Powell H, Murphy VE, Taylor DR, et al. Management of asthma in pregnancy guided by measurement of fraction of exhaled nitric oxide: a double-blind, randomised controlled trial. Lancet 2011; 378: 983-990.

25 Haldar P, Pavord ID, Shaw DE, et al. Cluster analysis and clinical asthma phenotypes. Am J Respir Crit Care Med 2008; 178: 218-224

26 Green RH, Brightling CE, McKenna S, et al. Asthma exacerbations and sputum eosinophil counts: a randomised controlled trial. Lancet 2002; 30: 1715-1721.

27 Agusti A, Fabbri LM, Singh D, et al. Inhaled corticosteroids in COPD: friend or foe? Eur Respir J 2018; $52: 1801219$.

28 Agusti A, Barbera JA, Wouters EF, et al. Lungs, bone marrow, and adipose tissue. A network approach to the pathobiology of chronic obstructive pulmonary disease. Am J Respir Crit Care Med 2013; 188: 1396-1406.

29 McDonald VM, Gibson PG, Scott HA, et al. Should we treat obesity in COPD? The effects of diet and resistance exercise training. Respirology 2016; 21: 875-882.

30 McDonald VM, Higgins I, Simpson JL, et al. The importance of clinical management problems in older people with COPD and asthma; do patients and physicians agree? Prim Care Respir J 2011; 20: 389-395.

31 McDonald VM, Higgins I, Wood LG, et al. Multidimensional assessment and tailored interventions for COPD: respiratory utopia or common sense? Thorax 2013; 68: 691-694.

32 Porsbjerg C, Menzies-Gow A. Co-morbidities in severe asthma: clinical impact and management. Respirology 2017; 22: 651-661

33 Tay TR, Hew M. Comorbid "treatable traits" in difficult asthma: current evidence and clinical evaluation. Allergy 2018; 73: 1369-1382

34 Chipps BE, Haselkorn T, Paknis B, et al. More than a decade follow-up in patients with severe or difficult-to-treat asthma: The Epidemiology and Natural History of Asthma: Outcomes and Treatment Regimens (TENOR) II. J Allergy Clin Immunol 2018; 141: 1590-1597.

35 Kerkhof M, Tran TN, Soriano JB, et al. Healthcare resource use and costs of severe, uncontrolled eosinophilic asthma in the UK general population. Thorax 2018; 73: 116-124.

36 Teague WG, Phillips BR, Fahy JV, et al. Baseline features of the Severe Asthma Research Program (SARP III) cohort: differences with age. J Allergy Clin Immunol Pract 2018; 6: 545-554.

37 Singh D, Kolsum U, Brightling CE, et al. Eosinophilic inflammation in COPD: prevalence and clinica characteristics. Eur Respir J 2014; 44: 1697-1700.

38 Shirtcliffe P, Weatherall M, Marsh S, et al. COPD prevalence in a random population survey: a matter of definition. Eur Respir J 2007; 30: 232-239.

39 van den Berge M, Aalbers R. The asthma-COPD overlap syndrome: how is it defined and what are its clinical implications? J Asthma Allergy 2016; 9: 27-35.

40 Boulet L-P, Phillips R, O’Byrne P, et al. Evaluation of asthma control by physicians and patients: comparison with current guidelines. Can Respir J 2002; 9: 417-423.

41 Clark VL, Gibson PG, Genn G, et al. Multidimensional assessment of severe asthma: a systematic review and meta-analysis. Respirology 2017; 22: 1262-1275

42 Shrimanker R, Choo XN, Pavord ID. A new approach to the classification and management of airways diseases: identification of treatable traits. Clin Sci 2017; 131: 1027-1043.

43 Woodcock J, LaVange LM. Master protocols to study multiple therapies, multiple diseases, or both. $N$ Engl J Med 2017; 377: 62-70.

44 Berry DA. The Brave New World of clinical cancer research: adaptive biomarker-driven trials integrating clinical practice with clinical research. Mol Oncol 2015; 9: 951-959.

45 Agusti A, Gea J, Faner R. Biomarkers, the control panel and personalized COPD medicine. Respirology 2016; 21: 24-33.

46 Murray CJL, Ezzati M, Flaxman AD, et al. GBD 2010: design, definitions, and metrics. Lancet 2012; 380 : 2063-2066.

47 Salomon JA, Vos T, Hogan DR, et al. Common values in assessing health outcomes from disease and injury: disability weights measurement study for the Global Burden of Disease Study 2010. Lancet 2012; 380: $2129-2143$.

48 Chowkwanyun M, Bayer R, Galea S. "Precision" public health - between novelty and hype. N Engl J Med 2018; 379: 1398-1400.

49 de Groot JC, Ten Brinke A, Bel EH. Management of the patient with eosinophilic asthma: a new era begins. ERJ Open Res 2015; 1: 00024-2015.

50 Petsky HL, Cates CJ, Kew KM, et al. Tailoring asthma treatment on eosinophilic markers (exhaled nitric oxide or sputum eosinophils): a systematic review and meta-analysis. Thorax 2018; 73: 1110-1119.

51 Beasley R, Hancox RJ, Harwood M, et al. Asthma and Respiratory Foundation NZ adult asthma guidelines: a quick reference guide. NZ Med J 2016; 129: 83-102.

52 Cousins JL, Wark PA, McDonald VM. Acute oxygen therapy: a review of prescribing and delivery practices. Int $J$ Chron Obstruct Pulmon Dis 2016; 11: 1067-1075.

53 Pretto JJ, McDonald VM, Wark PAB, et al. Multicentre audit of inpatient management of acute exacerbations of chronic obstructive pulmonary disease: comparison with clinical guidelines. Intern Med J 2012; 42: 380-387.

54 Spruit MA, Singh SJ, Garvey C, et al. An official American Thoracic Society/European Respiratory Society statement: key concepts and advances in pulmonary rehabilitation. Am J Respir Crit Care Med 2013; 188: e13-e64.

55 Coventry PA, Hind D. Comprehensive pulmonary rehabilitation for anxiety and depression in adults with chronic obstructive pulmonary disease: systematic review and meta-analysis. J Psychosom Res 2007; 63: 551-565.

56 Jacome C, Marques A. Pulmonary rehabilitation for mild COPD: a systematic review. Respir Care 2014; 59: 588-594.

57 Alison JA, McKeough ZJ, Johnston K, et al. Australian and New Zealand pulmonary rehabilitation guidelines Respirology 2017; 22: 800-819.

58 Rochester CL, Vogiatzis I, Holland AE, et al. An Official American Thoracic Society/European Respiratory society policy statement: enhancing implementation, use, and delivery of pulmonary rehabilitation. Am J Respir Crit Care Med 2015; 192: 1373-1386. 
59 McDonald VM, Vertigan AE, Gibson PG. How to set up a severe asthma service. Respirology 2011; 16: 900-911.

60 McDonald VM, Maltby S, Reddel HK, et al. Severe asthma: current management, targeted therapies and future directions - a roundtable report. Respirology 2017; 22: 53-60.

61 Rea H, McAuley S, Stewart A, et al. A chronic disease management programme can reduce days in hospital for patients with chronic obstructive pulmonary disease. Intern Med J 2004; 34: 608-614.

62 Burke H, Davis J, Evans S, et al. A multidisciplinary team case management approach reduces the burden of frequent asthma admissions. ERJ Open Res 2016; 2: 00039-2016.

63 Van der Meer V, Pasma H, Kempenaar-Okkema W, et al. A 1 day visit in a severe asthma centre: effect on asthma control. quality of life and health care use. Eur Respir J 2016; 48: 726-733.

64 Tay TR, Lee J, Radhakrishna N, et al. A structured approach to specialist-referred difficult asthma patients improves control of comorbidities and enhances asthma outcomes. J Allergy Clin Immunol Pract 2017; 5: 956-964.

65 MacDonald M, Korman T, King P, et al. Exacerbation phenotyping in chronic obstructive pulmonary disease. Respirology 2013; 18: 1280-1281.

66 Gibson PG, McDonald VM. Phenotyping asthma and COPD. BRN Rev 2016; 2: 239-252. 